\title{
Adaptive Limited Feedback Scheme for Stream Selection Based Interference Alignment in Heterogeneous Networks
}

\author{
Esra Aycan Beyazıt, Berna Özbek \\ Electrical and Electronics Engineering Department \\ Izmir Institute of Technology \\ İzmir, Turkey \\ \{esraaycan,bernaozbek\}@iyte.edu.tr
}

\author{
Didier Le Ruyet \\ CEDRIC/LAETITIA \\ Conservatoire National des Arts et Métiers (CNAM) \\ Paris, France \\ leruyet@cnam.fr
}

\begin{abstract}
This paper presents a stream selection based interference alignment approach with imperfect channel state information for heterogeneous networks. The proposed algorithm performs the selection of a stream sequence among a predetermined set of sequences. Those selected sequences are the ones that mostly contribute to the sum rate when performing the exhaustive search. These stream sequences form a regular structure where the first stream is associated to a pico user. The effect of imperfect channel state information on the proposed algorithm is analyzed and a bit allocation scheme is proposed by deriving an upper bound on the rate loss due to quantization.
\end{abstract}

\section{INTRODUCTION}

Heterogeneous networks provide a large number deployment of smaller cells with different transmit power levels under the coverage of the conventional macro cell. However, dense deployment of small cells increases the effect of the co-channel interference [1]. Interference alignment (IA) is one of the techniques to effectively mitigate interference in wireless networks [2]. It is introduced as a linear precoding technique that aligns the interfering signals in time, frequency, or space. Closed form solutions for the IA problem are difficult to obtain for large scale networks; therefore, for practical systems, iterative and distributed IA approaches have been intensively studied [3]. In addition, stream selection based approaches have been presented in [4] where the least interfering streams are selected to be in the null space of the previously selected ones. The IA approaches have been extended for cellular networks and heterogeneous networks [5] - [7].

The studies mentioned above assume that the channel state information (CSI) is available perfectly at all transmitters and receivers. Since this assumption is not realistic for practical systems, two methods have been implemented to provide the CSI, which are reciprocity and feedback. Since reciprocity is not possible to implement for frequency division duplexing (FDD) systems, feedback schemes have been commonly implemented in cellular networks. In the feedback mechanism, the quality of the CSI affects the performance of the IA. It has been shown that as the size of the codebook increases, the distortion caused by the limited feedback link decreases, but the feedback overhead increases in the network. Therefore, the number of bits for the quantization of the CSI should be optimized depending on the channel conditions [8].

In [9], an equal bit allocation has been studied on the stream selection based IA approach which selects multiple streams for each user comparing different distortion metrics for random vector quantization (RVQ). In the context of the heterogeneous networks, optimizing bit allocation can increase the performance of IA technique with the imperfect CSI by considering the distinctive features of the heterogeneous networks, such as unequal number of transmit antennas and transmit power levels [10] [11].

In this paper, we propose a novel stream selection based IA algorithm for the heterogeneous networks with imperfect channel state information. The algorithm starts by selecting a stream associated to the pico users in order to handle the interference coming from the macro base station (BS) to the pico user. The selection method is based on constructing a set of regular stream sequences. An adaptive feedback bit allocation is presented for the proposed algorithm to increase the system capacity for a fixed feedback load per user.

The rest of this paper is organized as follows. The system model and the channel quantization scheme are presented in Section II. The proposed algorithm is given in Section III. An adaptive bit allocation method is presented in Section IV. In Section $\mathrm{V}$, the performance evaluations are given and the study is concluded in Section VI.

\section{System MODEL}

In this study, a K-pair heterogeneous network composed of $K-1$ pico BSs and one macro BS with $N_{T_{k}}$ transmitter and $N_{R_{k}}$ receiver antennas is considered. For the sake of simplicity, macro BS - macro user pair is determined by the pair $k=1$, and pico BS - pico user pairs are kept in the set $\Gamma=\{2, \ldots, K\}$.

\section{A. Transmission Model}

The received signal at user $k$ is defined as follows.

$\mathbf{y}_{k}=\sqrt{P_{k}} \alpha_{k k} \mathbf{H}_{k k} \tilde{\mathbf{T}}_{k} \mathbf{s}_{k}+\sum_{\substack{j=1, j \neq k}}^{K} \sqrt{P_{j}} \alpha_{k j} \mathbf{H}_{k j} \tilde{\mathbf{T}}_{j} \mathbf{s}_{j}+\mathbf{n}_{k}$ 
where $\alpha_{k j} \mathbf{H}_{k j}$ is the channel matrix between the $j^{t h}$ transmitter and the $k^{t h}$ receiver with dimension $N_{R_{k}} \times N_{T_{j}}$. Each element of $\mathbf{H}_{k j}$ includes fading modeled as an independent and identically distributed complex Gaussian random variable with $\mathcal{C N}(0,1) . \alpha_{k j}$ denotes the pathloss and shadowing. For each receiver $k, \mathbf{n}_{k}$ is a $N_{R_{k}} \times 1$ vector. Each element of $\mathbf{n}_{k}$ represents additive white Gaussian noise with zero mean and variance of $\sigma^{2} . P_{k}$ is the transmit power of the $k^{t h} \mathrm{BS}$. $\tilde{\mathbf{T}}_{k}$ is the unitary precoding matrix of the $k^{t h}$ transmitter with dimension $N_{T_{k}} \times q_{k}$. The $k^{t h}$ transmitter has $q_{k}$ independent streams with $q_{k} \leq d_{k}$ where $d_{k}=\min \left(N_{R_{k}}, N_{T_{k}}\right)$. $\mathbf{s}_{k}$ is the symbol vector with dimension of $q_{k} \times 1$ and denoted as $\mathbf{s}_{k}=\left[s_{k, 1} \ldots s_{k, q_{k}}\right]^{T}$ where $\mathbb{E}\left[\left|s_{k, n}\right|^{2}\right]=1 / q_{k}, n=1, \ldots, q_{k}$.

Each user decodes the received signals by multiplying by the postcoding vectors, $\tilde{\mathbf{D}}_{k}$, of dimension $N_{R_{k}} \times q_{k}$. Thus, the decoded data symbols are obtained as $\hat{\mathbf{y}}_{k}=\tilde{\mathbf{D}}_{k}^{H} \mathbf{y}_{k}$. The data rate for the $i^{t h}$ stream of the $k^{t h}$ user can be expressed as follows.

$$
\tilde{\mathrm{R}}_{k i}=\log _{2}\left(1+\tilde{\gamma}_{k i}\right)
$$

where $\tilde{\gamma}_{k i}$ is the signal to interference noise ratio (SINR) for the $i^{\text {th }}$ stream of the $k^{t h}$ receiver and it is given by

$$
\tilde{\gamma}_{k i}=\frac{\left(P_{k} / q_{k}\right) \alpha_{k k}^{2}\left(\tilde{\mathbf{d}}_{k}^{i}\right)^{H} \mathbf{H}_{k k} \tilde{\mathbf{t}}_{k}^{i}\left(\tilde{\mathbf{t}}_{k}^{i}\right)^{H} \mathbf{H}_{k k}^{H} \tilde{\mathbf{d}}_{k}^{i}}{\left(\tilde{\mathbf{d}}_{k}^{i}\right)^{H} \tilde{\mathbf{B}}_{k i} \tilde{\mathbf{d}}_{k}^{i}}, \forall k, \forall i
$$

where $\tilde{\mathbf{t}}_{k}^{i}$ is the $i^{\text {th }}$ column vector of $\tilde{\mathbf{T}}_{k}$ with the size of $N_{T_{k}} \times 1$ and $\tilde{\mathbf{d}}_{k}^{i}$ is the $i^{t h}$ column vector of $\tilde{\mathbf{D}}_{k}$ with the size of $N_{R_{k}} \times 1$. The interference plus noise covariance matrix for the $i^{\text {th }}$ stream of the $k^{t h}$ receiver, $\tilde{\mathbf{B}}_{k i}$, is defined as

$$
\begin{aligned}
\tilde{\mathbf{B}}_{k i}= & \sum_{\substack{l=1, l \neq i}}^{q_{k}} \frac{P_{k}}{q_{k}} \alpha_{k k}^{2} \mathbf{H}_{k k} \tilde{\mathbf{t}}_{k}^{l} \tilde{\mathbf{t}}_{k}^{l H} \mathbf{H}_{k k}^{H}+ \\
& \sum_{\substack{j=1 \\
j \neq k}}^{K} \sum_{q=1}^{q_{j}} \frac{P_{j}}{q_{j}} \alpha_{k j}^{2} \mathbf{H}_{k j} \tilde{\mathbf{t}}_{j}^{q} \tilde{\mathbf{t}}_{j}^{q H} \mathbf{H}_{k j}^{H}+\sigma^{2} \mathbf{I}_{N_{R_{k}}}, \forall k, \forall i
\end{aligned}
$$

The achievable sum rate is calculated as follows.

$$
\tilde{\mathrm{SR}}=\sum_{k=1}^{K} \sum_{i=1}^{q_{k}} \tilde{\mathrm{R}}_{k i}
$$

The main objective is to mitigate the interference while finding the best stream sequence and guaranteeing at least one stream for each user under the quantized feedback link. The main objective can be formulated as follows.

$$
\begin{gathered}
\left\{\left(\tilde{\mathbf{T}}_{k}^{*}, \tilde{\mathbf{D}}_{k}^{*}\right)\right\}_{k=1, \ldots, K}=\underset{\tilde{\mathbf{T}}_{k}, \tilde{\mathbf{D}}_{k}}{\operatorname{argmax}} \tilde{\mathrm{SR}} \\
\text { s.t. } \quad q_{k} \geq 1 \quad k=1, \ldots, K
\end{gathered}
$$

\section{B. Channel Quantization Model}

In this section, a limited feedback scheme is given based on RVQ. The proposed IA algorithm requires all CSI to compute all precoding and postcoding vectors. Therefore, a centralized feedback model in which the macro BS collects all CSI from pico BSs through the delay free backhaul links is considered. For the distortion metrics, each codebook contains $2^{B_{k j}}$ codewords which are randomly generated, where $B_{k j}$ is the number of quantization bits to quantize the channel between the $j^{t h}$ transmitter and the $k^{t h}$ receiver. The codewords are independent and isotropically distributed over the unit sphere. It is assumed that the CSI has been perfectly known at each receiver as $\mathbf{H}_{k j}=\overline{\mathbf{H}}_{k j} \times\left\|\mathbf{H}_{k j}\right\|_{F}$ where $\overline{\mathbf{H}}_{k j}$ is the CDI. We perform quantization for CDI using Chordal distance [12].

The quantization error caused by RVQ can be modeled as $\overline{\mathbf{h}}_{k j}=\sqrt{1-e_{k j}} \tilde{\mathbf{h}}_{k j}+\sqrt{e_{k j}} \mathbf{z}_{k j}$ where $\mathbf{z}_{k j}$ is the unit vector representing the direction of the quantization error vector and is isotropically distributed in the null space of $\overline{\mathbf{h}}_{k j}$. $e_{k j}$ is the minimum of $2^{N_{d}}$ independent $\beta\left(\left(N_{T_{j}} N_{R_{k}}-1\right), 1\right)$ random variables [16]. The quantization error can be modeled in the matrix form as $\overline{\mathbf{H}}_{k j}=\sqrt{1-e_{k j}} \tilde{\tilde{\mathbf{H}}}_{k j}+\sqrt{e_{k j}} \mathbf{Z}_{k j}$ where $\mathbf{Z}_{k j} \in$ $\mathbb{C}^{N_{R_{k}} \times N_{T_{j}}}$ is the reshaped matrix of vector $\mathbf{z}_{k j} \in \mathbb{C}^{N_{R_{k}} N_{T_{j}} \times 1}$.

\section{Restricted Advanced Successive Null Space STREAM SELECTION}

The proposed successive null space stream selection algorithm (RASNSSS) is developed by analyzing the data collected from extensive exhaustive searches. It performs the selection of a stream sequence among a predetermined set of sequences in order to reduce the complexity while satisfying the constraint given in Eq. (6b). This predetermined set is composed of the sequences with the highest probability of occurrence while performing the exhaustive search with the quantized CSI. The construction of the stream sequences based on the regular structure is expressed as follows.

Streams are identified using the singular values which are computed from the SVD of all the quantized CSIs, $\left(\alpha_{k k} \tilde{\mathbf{H}}_{k k}\right)=\tilde{\mathbf{U}}_{k} \tilde{\mathbf{S}}_{k} \tilde{\mathbf{V}}_{k}^{H}$ and each stream $i$ can be expressed as $\pi_{i}=\left(k_{i}, l_{i}\right)$ where $k_{i} \in\{1, \ldots, K\}, l_{i} \in\left\{1, \ldots, q_{k_{i}}\right\}$ and $i \in$ $\{1, \ldots, r\}$. The set of all possible stream sequences can be defined as $\Phi=\Phi_{1} \cup \ldots \cup \Phi_{j} \cup \ldots \cup \Phi_{r}$ where $\Phi_{j}$ is the set of all permutations of length $j \in\{1, \ldots, r\}$ given by

$$
\Phi_{j}=\left\{\pi=\left(\pi_{1} \pi_{2} \ldots \pi_{j}\right) \mid \forall i, i^{\prime} \in\{1, \ldots, j\}, \pi_{i} \neq \pi_{i^{\prime}} \text { if } i \neq i^{\prime}\right\}
$$

All stream sequences that include at least one stream from each BS-user pair are kept in set $\Pi$ which can be defined as follows.

$$
\begin{aligned}
\Pi= & \left\{\pi=\left(\pi_{1} \pi_{2} \ldots \pi_{j}\right) \mid \pi \in \Phi_{j} ; j \geq K ;\right. \\
& \left.\forall k, \exists m \in\{1, \ldots, j\} \text { such that } k_{m}=k\right\}
\end{aligned}
$$

Generated stream sequences are kept in set $\Pi_{A}$ [12]. The algorithm given in [12] can continue to select streams after the construction of $\Pi_{A}$, because perfect CSI is assumed to be available at both transmitters and receivers and, thus, it is possible to increase the sum-rate further by adding more streams to the constructed stream sequences. However, in the case of limited feedback as considered in this study, continuing to select more streams generally causes a degradation in the sum rate due to the quantization. Therefore, the proposed algorithm is the restricted version of the algorithm in [12].

To explain the construction of $\Pi_{A}$, each pico user is associated with a series of stream sequence sets. More precisely, the following sets are constructed for each pico user $k^{\prime} \in \Gamma$. 
- $\Xi_{k^{\prime}}=\left\{\pi=\left(\pi_{1} \pi_{2} \ldots \pi_{d_{k^{\prime}}}\right) \mid \pi \in \Phi ; \forall i \in\left\{1, \ldots, d_{k^{\prime}}\right\}\right.$,

$\pi_{i}=\left(k^{\prime}, l_{i}\right)$ for some $\left.l_{i} \in\left\{1, \ldots, d_{k^{\prime}}\right\}\right\}$

- $\Upsilon_{k^{\prime}, h^{\prime}}=\left\{\pi=\left(\pi_{1} \pi_{2} \ldots \pi_{|\Gamma|-2}\right) \mid \pi \in \Phi ; k_{i} \in \Gamma \backslash\left\{k^{\prime}, h^{\prime}\right\}\right.$,

$\forall i \in\{1, \ldots,|\Gamma|-2\}$, and $k_{i} \neq k_{j}$ if $\left.i \neq j\right\}$

- $\Lambda=\{p \mid p=(k, l)$ and $k, l=1\}$

- $\Delta_{h^{\prime}}=\left\{p \mid p=\left(h^{\prime}, l\right)\right.$ and $\left.l=1\right\}$

Based on the above sets, $\Pi_{A}$ is constructed as follows.

$$
\Pi_{A}=\bigcup_{k^{\prime} \in \Gamma} \Xi_{k^{\prime}} \times\left(\bigcup_{h^{\prime} \in \Gamma \backslash\left\{k^{\prime}\right\}} \Upsilon_{k^{\prime}, h^{\prime}} \times \Lambda \times \Delta_{h^{\prime}}\right)
$$

The whole procedure of the RASNSSS is explained in Alg. 1.

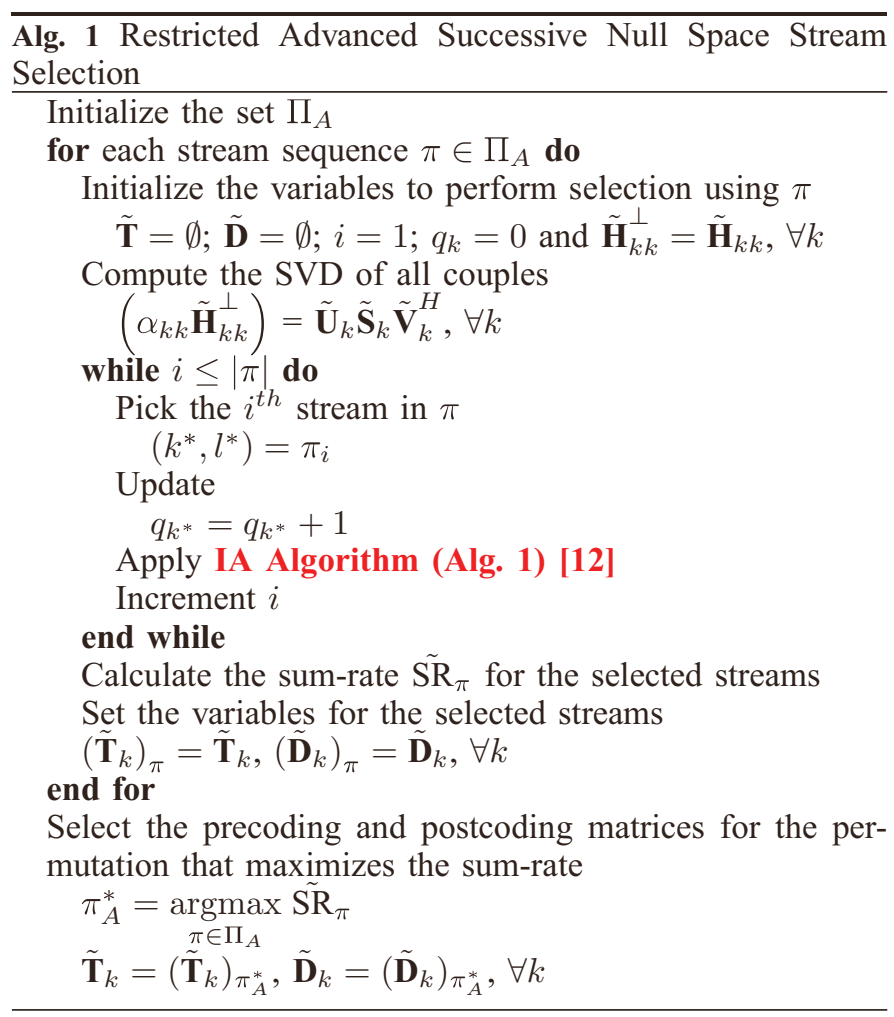

\section{The Proposed Bit Allocation Method}

The main objective of the adaptive feedback bit allocation is to maximize the average sum rate by optimizing the number of bits for each user to quantize the macro and pico CDIs. A certain number of bits to quantize the CDIs is adaptively and locally allocated to each user. The optimization problem of the bit allocation for RASNSSS can be formulated for each user as follows.

$$
\begin{aligned}
& \max _{B_{k j} ; j=1, \ldots, K} \mathbb{E}\left[\tilde{\mathrm{R}}_{k i}\right] \\
& \text { s.t. } \sum_{j=1}^{K} B_{k j} \leq B_{k}
\end{aligned}
$$

The bit allocation problem in Eq.(14) is considered for the high SINR region where $\log _{2}(1+x) \approx \log _{2}(x)$, since the interference is mitigated by performing the RASNSSS. Furthermore, the interfering and the desired channel terms are assumed to be independently distributed random variables. Therefore, $\mathbb{E}\left[\tilde{\mathrm{R}}_{k i}\right]$ can be rewritten by using Eq. (3) as follows.

$$
\begin{aligned}
& \mathbb{E}[\underbrace{\log _{2}\left(\left(P_{k k} / q_{k}\right)\left(\tilde{\mathbf{d}}_{k}^{i}\right)^{H} \mathbf{H}_{k k} \tilde{\mathbf{t}}_{k}^{i} \tilde{\mathbf{t}}_{k}^{i H} \mathbf{H}_{k k}^{H} \tilde{\mathbf{d}}_{k}^{i}\right)}_{a}]- \\
& \mathbb{E}[\underbrace{\log _{2}\left(\sum_{\substack{l=1, l \neq \neq i}}^{q_{k}}\left(P_{k k} / q_{k}\right)\left(\tilde{\mathbf{d}}_{k}^{l}\right)^{H} \mathbf{H}_{k k} \tilde{\mathbf{t}}_{k}^{l} \tilde{\mathbf{t}}_{k}^{l} \mathbf{H}_{k k}^{H} \tilde{\mathbf{d}}_{k}^{l}+\right.}_{b} \\
& \underbrace{\left.\left.\sum_{\substack{j=1 \\
j \neq k}}^{K} \sum_{q=1}^{q_{j}}\left(P_{k j} / q_{j}\right)\left(\tilde{\mathbf{d}}_{j}^{q}\right)^{H} \mathbf{H}_{k j} \tilde{\mathbf{t}}_{j}^{q} \tilde{\mathbf{t}}_{j}^{q H} \mathbf{H}_{k j}^{H} \tilde{\mathbf{d}}_{j}^{q}\right)\right]}_{b}
\end{aligned}
$$

where $P_{k j}$ is the received power at the $k^{\text {th }}$ user from the $j^{\text {th }}$ BS and it is calculated as $P_{k j}=P_{j} \alpha_{k j}^{2}, \forall k, \forall j$. Therefore, the first term of Eq. (15) can be rewritten as follows [13].

$$
a=\log _{2}\left(\left(P_{k k} / q_{k}\right)\left\|\mathbf{H}_{k k}\right\|_{F}^{2}\left(\left(1-e_{k k}\right)\left|\left(\tilde{\mathbf{d}}_{k}^{i}\right)^{H} \tilde{\mathbf{H}}_{k k} \tilde{\mathbf{t}}_{k}^{i}\right|^{2}\right)\right)
$$

Considering the $\left|\left(\tilde{\mathbf{d}}_{k}\right)^{H} \tilde{\overline{\mathbf{H}}}_{k j} \tilde{\mathbf{t}}_{j}\right|$ terms are approximately zero due to the IA scheme and using the relation $|x+y|^{2} \leq(|x|+$ $|y|)^{2}$, the second term of Eq. (15), $b$, can be written as follows.

$$
\begin{gathered}
b \leq \log _{2}\left(\sum_{\substack{l=1, l \neq i}}^{q_{k}}\left(P_{k k} / q_{k}\right)\left\|\mathbf{H}_{k k}\right\|_{F}^{2}\left(e_{k k}\left|\left(\tilde{\mathbf{d}}_{k}^{i}\right)^{H} \mathbf{Z}_{k k} \tilde{\mathbf{t}}_{k}^{l}\right|^{2}\right)+\right. \\
\left.\sum_{\substack{j=1 \\
j \neq k}}^{K} \sum_{q=1}^{q_{j}}\left(P_{k j} / q_{j}\right)\left\|\mathbf{H}_{k j}\right\|_{F}^{2}\left(e_{k j}\left|\left(\tilde{\mathbf{d}}_{k}^{i}\right)^{H} \mathbf{Z}_{k j} \tilde{\mathbf{t}}_{j}^{q}\right|^{2}\right)\right)
\end{gathered}
$$

Using Jensen's inequality, the upper bound for Eq. (15) can be obtained as follows.

$\mathbb{E}[a]-\mathbb{E}[b] \leq$

$$
\begin{aligned}
& \log _{2}(\underbrace{\left.\mathbb{E}\left[\left(P_{k k} / q_{k}\right)\left\|\mathbf{H}_{k k}\right\|_{F}^{2}\left(\left(1-e_{k k}\right)\left|\left(\tilde{\mathbf{d}}_{k}^{i}\right)^{H} \tilde{\mathbf{H}}_{k k} \tilde{\mathbf{t}}_{k}^{i}\right|^{2}\right)\right]\right)}_{T 1}- \\
& \log _{2}(\underbrace{\sum_{\substack{l=1, l \neq i}}^{q_{k}} \mathbb{E}\left[\left(P_{k k} / q_{k}\right)\left\|\mathbf{H}_{k k}\right\|_{F}^{2}\left(e_{k k}\left|\left(\tilde{\mathbf{d}}_{k}^{i}\right)^{H} \mathbf{Z}_{k k} \tilde{\mathbf{t}}_{k}^{l}\right|^{2}\right)\right]}_{T 2}+ \\
& \underbrace{\left.\sum_{\substack{j=1 \\
j \neq k}}^{K} \sum_{q=1}^{q_{j}} \mathbb{E}\left[\left(P_{k j} / q_{j}\right)\left\|\mathbf{H}_{k j}\right\|_{F}^{2}\left(e_{k j}\left|\left(\tilde{\mathbf{d}}_{k}^{i}\right)^{H} \mathbf{Z}_{k j} \tilde{\mathbf{t}}_{j}^{q}\right|^{2}\right)\right]\right)}_{T 3}
\end{aligned}
$$

Since $\mathbb{E}\left[\left\|\mathbf{H}_{k k}\right\|_{F}^{2}\right]=N_{T_{k}} N_{R_{k}}$ [13], the first term of Eq. (18), $T 1$ can be expressed as follows [14].

$$
T 1 \leq\left(P_{k k} / q_{k}\right)\left(1-2^{-w}\right)
$$

where $w=\frac{B_{k k}}{N_{T_{k}} N_{R_{k}}-1}$. The second and the third term of Equation (18), $T 2$ and $T 3$, can be expressed as follows [15] [16].

$$
T 2+T 3 \leq \sum_{\substack{l=1, l \neq i}}^{q_{k}}\left(P_{k k} / q_{k}\right) 2^{-w}+\sum_{\substack{j=1 \\ j \neq k}}^{K} P_{k j} 2^{-y}
$$


where $y=\frac{B_{k j}}{N_{T_{j}} N_{R_{k}}-1}$. Using Eq. (19) and Eq. (20) in Eq. (15), the optimization problem can be expressed as follows.

$$
\begin{aligned}
& \max _{\substack{B_{k j} ; \\
j=1, \ldots, K}}\left[\log _{2}\left(\left(P_{k k} / q_{k}\right)\left(1-2^{-w}\right)\right)-\right. \\
& \left.\log _{2}\left(\sum_{\substack{l=1, l \neq i}}^{q_{k}}\left(P_{k k} / q_{k}\right) 2^{-w}+\sum_{\substack{j=1 \\
j \neq k}}^{K} P_{k j} 2^{-y}\right)\right] \\
& \text { s.t. } \sum_{j=1}^{K} B_{k j} \leq B_{k}
\end{aligned}
$$

Solutions for the problem expressed in Equation (21) are obtained by using an optimization software tool [17]. After obtaining the $B_{k j}$ values which are real numbers, a round operation is applied to get integer values.

\section{Performance Results}

The performance of the RASNSSS is evaluated in a heterogeneous network as illustrated in Figure 1. The number of transmit antennas is 2 for each pico cell and 4 for the macro cell. Each cell has one user randomly placed inside the cell coverage. Each user has 2 receive antennas. Locations of the pico cells are identified by the ratio $d / R$ where $R$ is the macro cell radius and $d$ is the distance between the macro BS and pico BSs. Since, in practice, pico cells are generally deployed closer to the cell edge of the macro cells, the ratio ranges from 0.6 to 1. In addition, the distance between the pico cells is constant at $L=200 \mathrm{~m}$. Simulations are carried out by using the system

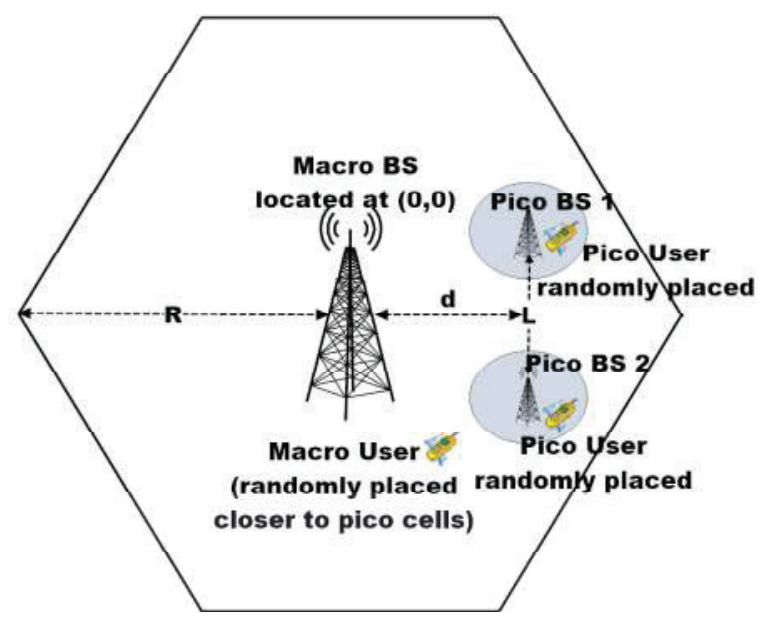

Fig. 1. Picocells are symmetrically deployed

parameters listed in Table I. In Figure 2, the performance

TABLE I. System PARAMETERS

\begin{tabular}{|c|c|}
\hline Parameter Name & Parameter Value \\
\hline \hline Transmit Power & $43 \mathrm{dBm}($ macro), $24 \mathrm{dBm}(\mathrm{pico})$ \\
\hline Bandwidth & $10 \mathrm{MHz}$ \\
\hline Carrier Frequency & $2.1 \mathrm{GHz}$ \\
\hline Noise Power & $-174 \mathrm{dBm} / \mathrm{Hz}$ \\
\hline Cell Radius & $1000 \mathrm{~m}($ makro), $100 \mathrm{~m}($ piko $)$ \\
\hline Path loss (macro) & $L_{p}=128.1+37.6 \log 10(R(\mathrm{~km})) \mathrm{dB}$ \\
\hline Path loss (pico) & $L_{p}=140.7+36.7 \log 10(R(\mathrm{~km})) \mathrm{dB}$ \\
\hline Shadowing std. dev. & $8 \mathrm{~dB}($ macro), $10 \mathrm{~dB}(\mathrm{pico})$ \\
\hline
\end{tabular}

comparison of different bit allocation schemes is given for the total number of feedback bits $B_{T}=\sum_{k=1}^{K} B_{k}=63$. For the limited feedback scheme, there are 9 channels in the scenario which includes the desired channels and the interfering channels. Therefore, the number of allocated bits to each channel is 7 in the equal bit allocation (EBA) scheme. It can be observed that the proposed adaptive feedback bit allocation given in Eq. (21) outperforms the EBA scheme. In addition, different bit allocation schemes are compared for RASNSSS algorithm by allocating different number of bits to each user, such as $B_{1}=5, B_{2}=29, B_{3}=29$ (OPT1), and $B_{1}=21, B_{2}=21, B_{3}=21$ (OPT2). It is observed that the performance increases as more bits are allocated to the pico users. The interference channels between the pico users and the macro BS require higher number of bits, since the generated interference is very strong.

Furthermore, the performance results indicate that RASNSSS outperforms the existing stream selection approaches such as ESNSSS [4] and SNSSS [4], by getting closer to the upper bound set by the exhaustive search for the OPT1 bit allocation scheme as shown in Figure 2. In addition, the performances of the max-SINR and the min-Leak algorithms [3] are compared for the single stream transmission and RASNSSS achieves higher performance even when a pico cell has more than one stream.

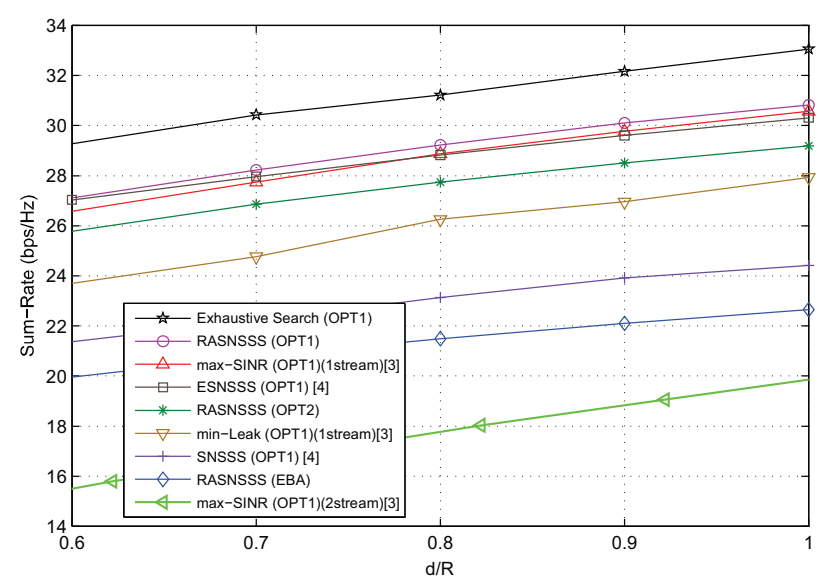

Fig. 2. Performance comparison for adaptive bit allocation with $B_{T}=63$.

\section{CONCLUSION}

In this study, we have proposed the RASNSSS algorithm for the limited feedback schemes in the heterogeneous networks and an adaptive bit allocation has been presented to reduce the quantization error on the proposed algorithm. It has been observed that more bits should be allocated to the interference channels between the macro BS and pico users since the generated interference by the macro BS to the pico users is very dominant. 


\section{REFERENCES}

[1] H. Zhang; S. Chen; X. Li; H. Ji; X. Du, "Interference management for heterogeneous networks with spectral efficiency improvement," IEEE Wireless Communications 2015, vol.22, no.2, pp.101-107.

[2] S. A. Jafar, "Interference Alignment: A New Look at Signal Dimensions in a Communication Network," Foundations and Trends in Communications and Information Theory 2011, vol. 7, no. 1, pp. 1-136.

[3] K. Gomadam; V.R. Cadambe; S. A. Jafar, "A Distributed Numerical Approach to Interference Alignment and Applications to Wireless Interference Networks," IEEE Transactions on Information Theory 2011, vol.57, no.6, pp.3309-3322.

[4] M. Amara; M. Pischella; D. Le Ruyet, "Enhanced stream selection for sum-rate maximization on the interference channel," International Symposium on Wireless Communication Systems (ISWCS) Paris, 2012, pp. 151-155.

[5] C. Suh; M. Ho; D. N. C. Tse, "Downlink Interference Alignment," IEEE Transactions on Communications 2011, vol.59, no.9, pp.2616-2626.

[6] G. Liu; M. Sheng; X. Wang; W. Jiao; Y. Li; J. Li, "Interference Alignment for Partially Connected Downlink MIMO Heterogeneous Networks," IEEE Transactions on Communications 2015, vol.63, no.2, pp.551-564.

[7] E. Aycan; B. Özbek; D. Le Ruyet, "Hierarchical successive stream selection for heterogeneous network interference," IEEE Wireless Communications and Networking Conference (WCNC) Istanbul, 2014, pp.11431148 .

[8] B. Özbek; D. Le Ruyet, Feedback strategies for wireless communication systems. Springer-Engineering Series Book, Springer Science Business Media New York, U.S.A, 2014

[9] E. Aycan; B. Özbek; D. Le Ruyet, "Improved Successive Stream Selection with Quantized Channel in Heterogeneous Networks," in International Symposium on Wireless Communication Systems ISWCS Brussels, 2015, pp. 606-610.

[10] M. Rihan; M. Elsabrouty; O. Muta; H. Furukawa, "Interference Alignment with Limited Feedback for Macrocell-Femtocell Heterogeneous Networks," IEEE Vehicular Technology Conference (VTC) Glasgow, 2015, pp. 1-5.

[11] Q. Niu; Z. Zeng; T. Zhang; Q. Gao; S. Sun, "Interference Alignment and Bit Allocation in Heterogeneous Networks with Limited Feedback," IEEE International Symposium on Wireless Personal Multimedia Communications (WPMC) Sydney, NSW, 2014, pp. 514-519.

[12] E. Aycan Beyazit; B. Özbek; D. Le Ruyet, "On Stream Selection for Interference Alignment in Heterogeneous Networks," EURASIP Journal on Wireless Communications and Networking 2016, pp.0-18, DOI: 10.1186/s13638-016-0575-7.

[13] K. Anand; E. Gunawan; Y. L. Guan, "Beamformer Design for the MIMO Interference Channels under Limited Channel Feedback," IEEE Transactions on Communications 2013, vol.61, no.8, pp.3246-3258.

[14] B. Özbek; D. Le Ruyet, "Adaptive limited feedback links for cooperative multi-antenna multicell networks," EURASIP Journal on Wireless Communications and Networking 2014, vol. 2014, p. 193.

[15] N. Ravindran; N. Jindal, "Limited feedback-based block diagonalization for the MIMO broadcast channel," IEEE Journal on Selected Areas in Communications 2008, vol.26, no.8, pp.1473-1482.

[16] N. Jindal, "MIMO Broadcast Channels With Finite-Rate Feedback," IEEE Transactions on Information Theory 2006, vol.52, no.11, pp.50455060 .

[17] M. Grant; S. Boyd, Mar. 2014. CVX: Matlab software for disciplined convex programming, version 2.1 . http://cvxr.com/cvx. 